\title{
An Integrated Safety Analysis of Infants and Children with Symptomatic Spinal Muscular Atrophy (SMA) Treated with Nusinersen in Seven Clinical Trials
}

\author{
Basil T. Darras ${ }^{1} \cdot$ Michelle A. Farrar $^{2} \cdot$ Eugenio Mercuri $^{3} \cdot$ Richard S. Finkel $^{4} \cdot$ Richard Foster $^{5} \cdot$ Steven G. Hughes $^{6}$. \\ Ishir Bhan ${ }^{7}(1)$. Wildon Farwell ${ }^{7}$ Sarah Gheuens ${ }^{7}$
}

Published online: 16 August 2019

(c) The Author(s) 2019

\begin{abstract}
Background Treatment with nusinersen has demonstrated significant and clinically meaningful benefits in clinical trials in infants and children with spinal muscular atrophy (SMA).

Objective The objective of this analysis was to characterize the safety of nusinersen across the clinical trial program in infants and children with symptomatic SMA.

Methods An integrated safety analysis evaluated end of study data from seven completed clinical trials that enrolled infants and children with symptomatic SMA who were treated with intrathecal nusinersen or underwent sham procedures. Two of the studies were conducted in symptomatic infants with infantile-onset SMA (most likely to develop SMA type I or II) and the remaining five in symptomatic children and adolescents with later-onset SMA (have or are most likely to develop SMA type II or III). Safety assessments included incidence of adverse events (AEs), physical and neurological examinations, vital signs, clinical laboratory tests (serum chemistry, hematology, and urinalysis), and electrocardiograms.

Results Data were analyzed from 323 infants and children, including 240 treated with nusinersen (100 with infantile-onset SMA and 140 with later-onset SMA) and 83 who underwent sham procedures (41 infantile-onset, 42 later-onset). Median (range) exposure to nusinersen was 449.0 (6-1538) days (375.9 participant-years). The most common AEs with nusinersen were pyrexia, upper respiratory tract infection, nasopharyngitis, vomiting, headache, and constipation. The incidence of serious AEs was lower with nusinersen than with the sham procedure ( $41 \%$ vs. $61 \%$ ). The overall incidence of respiratory, thoracic, and mediastinal AEs was higher in participants with symptomatic infantile-onset SMA than those with symptomatic later-onset SMA and similar in nusinersen- versus sham procedure-treated participants. Rates of post-lumbar puncture syndrome and related events were higher with nusinersen versus sham procedure in later-onset SMA participants. No abnormal patterns or trends in laboratory test results were observed.

Conclusions Nusinersen demonstrated a favorable safety profile in children with symptomatic infantile- and later-onset SMA. Most reported AEs and serious AEs were consistent with the nature and frequency of events typically seen with SMA or in the context of lumbar puncture procedures.
\end{abstract}

Registration NCT01494701, NCT01703988, NCT01839656, NCT02193074, NCT02292537, NCT01780246, NCT02052791.

Electronic supplementary material The online version of this article (https://doi.org/10.1007/s40263-019-00656-w) contains supplementary material, which is available to authorized users.

Ishir Bhan

Ishir.Bhan@biogen.com

Extended author information available on the last page of the article 


\section{Key Points}

A smaller proportion of children with symptomatic spinal muscular atrophy (SMA) treated with nusinersen compared with sham procedure experienced serious adverse events (AEs).

No pattern of laboratory abnormalities was observed, including no cases meeting criteria for Hy's Law cases or with evidence of liver failure.

The nature and frequency of most reported AEs and serious AEs were consistent with events typically seen with SMA or in the context of lumbar puncture procedures.

In this analysis, there was no evidence that nusinersen treatment led to thrombocytopenia or hepatic or renal impairment, toxicities that have been reported with other antisense oligonucleotides.

\section{Introduction}

Spinal muscular atrophy (SMA) is an autosomal recessive neuromuscular disorder that is characterized by progressive and often severe muscular atrophy and weakness [1]. The disorder is one of the leading causes of infant mortality and is estimated to occur in $8.5-10.3$ per 100,000 live births [2-5]. SMA is caused by homozygous deletion or mutation in the survival motor neuron 1 (SMNI) gene, resulting in decreased levels of SMN protein expression and degeneration of motor neurons in the anterior horn cells of the spinal cord and brain stem $[1,6]$. The homologous SMN2 gene also codes for the SMN protein but fails to fully compensate for SMN1 gene loss due to aberrant splicing, resulting in insufficient amounts of SMN protein [1].

SMA has been broadly classified into four main phenotypes [1]. In SMA type I, symptoms first appear at or before 6 months of age and can include severe generalized hypotonia, weakness of limbs and neck, areflexia, tongue fasciculations, abdominal paradoxical breathing, and respiratory failure $[1,7]$. Affected individuals never sit independently, require more intensive and supportive care than those with less severe forms of SMA, and generally have a lifespan of $<2$ years if not treated $[1,7]$. Those with symptom onset after 6 months of age but before adulthood are considered to have later-onset SMA and are most likely to develop SMA type II or III, depending on the age of symptom onset and highest motor function achieved [1, 7]. Children with SMA type II begin displaying symptoms at 7-18 months of age; these can include mild to moderate hypotonia, weakness, areflexia, and hand tremor. Children with SMA type II develop the ability to sit independently and may stand with support, but they are never able to walk independently and often develop significant restrictive lung disease $[1,7,8]$. SMA type III symptoms emerge at $>18$ months of age and are heterogeneous. Individuals with type III typically reach independent walking, but some require wheelchair assistance for mobility in childhood $[1,7,8]$. The legs are often more severely affected than the arms [1]. Type IV develops in adulthood and is the least severe form of SMA [1]. Management of SMA, particularly the more severe forms, requires a multidisciplinary approach due to the nature of the symptoms. Most importantly, pulmonary support is frequently necessary in patients with type I and II SMA, as respiratory failure is the most common cause of mortality. Due to respiratory muscle weakness, patients are often unable to maintain a clear airway, require ventilatory support, and are at risk of pneumonia and other respiratory tract infections and respiratory distress $[1,9,10]$.

The historical SMA classifications described above are rapidly evolving with the advent of effective SMA treatment options. Nusinersen is an antisense oligonucleotide (ASO) that modifies the splicing of SMN2 pre-messenger RNA, resulting in increased levels of full-length SMN protein [11]. Treatment with nusinersen has demonstrated significant and clinically meaningful benefits on motor function in infants and children with SMA [12-16]. The objective of this safety analysis is to characterize the safety of nusinersen across the clinical trial program in symptomatic infants and children with SMA.

\section{Methods}

\subsection{Study Design and Participants}

This integrated safety analysis evaluated end of study data from symptomatic infants and children with SMA who underwent administration of nusinersen via intrathecal injection or a sham procedure (consisting of a small needle prick to the lower back, which was then covered with a bandage) in seven completed clinical trials (five open-label, two blinded; Fig. 1). This integrated analysis was performed on data from trials in symptomatic infants and does not include the NURTURE study (NCT0286553) of nusinersen treatment in presymptomatic infants; we believe the natural history, and therefore the AE profile, is markedly different in individuals who initiate nusinersen after symptom onset compared with those who initiate treatment before symptom onset. Of the included studies, two were in infantileonset SMA (most likely to develop SMA type I or II), as participants were aged 7 months or younger at screening, 
Fig. 1 Overview of the clinical studies used for the integrated analyses. SMA spinal muscular atrophy
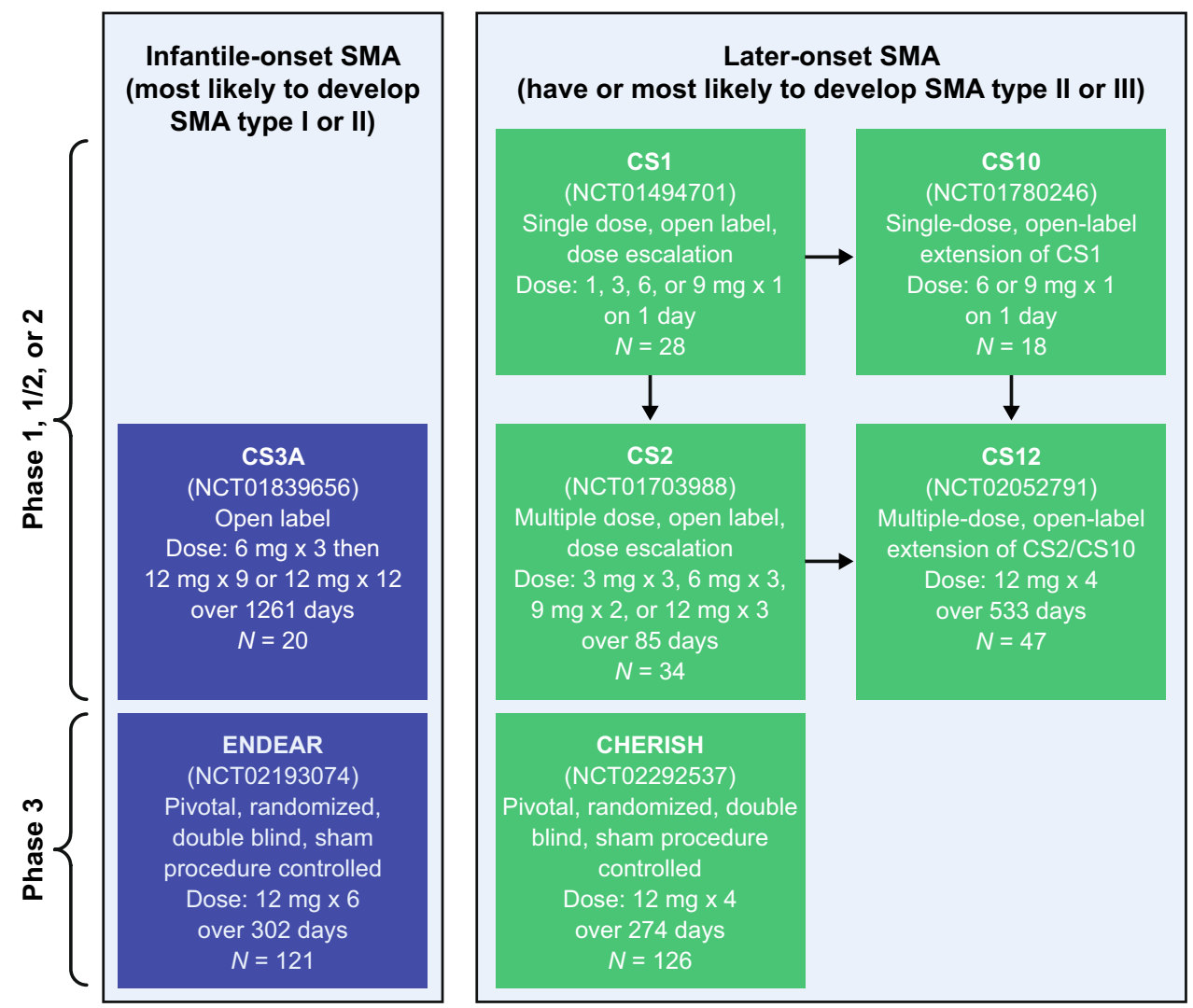

had onset of SMA symptoms at or before 6 months of age, and had SMN1 homozygous gene deletion or mutation [12, 13]. In CS3A (NCT01839656), an open-label, escalatingdose, phase 2 study, participants received either three 6-mg doses followed by nine $12-\mathrm{mg}$ doses $(n=4)$, or twelve $12-\mathrm{mg}$ doses $(n=16)$, of nusinersen and were followed for 1352 days (data cutoff date August 21, 2017). In the other study, ENDEAR (NCT02193074), a randomized, doubleblind, sham-controlled, phase 3 study, participants received up to six 12-mg equivalent doses of nusinersen $(n=80)$ or sham procedures $(n=41)$ over 302 days; the study follow-up extended to day 394, but the data collected are up to the last study visit (December 16, 2016) due to early termination of the study [12].

The remaining five trials were in children and adolescents with later-onset symptomatic SMA (i.e., those who have or are most likely to develop SMA type II or III). In CS1 (NCT01494701), a phase 1, multicenter, open-label, dose-escalation study in children aged 2-14 years with an SMN1 homozygous gene deletion, a single 1-, 3-, or 6-mg dose was administered to six participants each and a 9-mg dose was administered to 10 participants $[16,17]$. Eighteen of the 22 participants in $\mathrm{CS} 1$ who received $\mathrm{a} \geq 3$-mg dose enrolled in the open-label extension, CS10 (NCT01780246), in which a single $6-\mathrm{mg}(n=4)$ or $9-\mathrm{mg}(n=14)$ dose of nusinersen was administered. Those who received a $1-\mathrm{mg}$ dose in CS1 ( $n=6)$ could enroll in CS2 (NCT01703988), a multidose, open-label, dose-escalation study in which participants aged 2-15 years with an SMN1 homozygous gene deletion or mutation received either two doses of nusinersen $9 \mathrm{mg}(n=9)$, or three doses of nusinersen $3 \mathrm{mg}$ $(n=8), 6 \mathrm{mg}(n=8)$, or $12 \mathrm{mg}(n=9)$, over 85 days. CS2 also enrolled treatment-naïve participants $(n=28)$. In CS12 (NCT02052791), a multidose, open-label extension study of CS2 and CS10, participants $(n=47)$ received four 12-mg doses over 533 days [15]. Participants in CS1, CS2, and CS10 who continued treatment in CS12 were followed up to the last study visit of CS12 (January 24, 2017). In CHERISH (NCT02292537), a multicenter, double-blind, shamcontrolled, phase 3 trial, participants aged 2-12 years with homozygous deletion, mutation, or compound heterozygote in SMN1 and symptom onset after 6 months of age received up to four 12-mg doses ( $n=84)$ or underwent sham procedures $(n=42)$ over 274 days; the study follow-up extended to day 456 , but the trial was stopped following a prespecified interim analysis, and the data collected are up to the last study visit (February 20, 2017) [14].

\subsection{Safety and Tolerability Assessments}

Safety assessments included adverse events (AEs), physical and neurological examinations, vital signs, clinical 
laboratory tests (serum chemistry, hematology, and urinalysis), and electrocardiograms (ECGs). An AE was defined as any untoward medical occurrence temporally associated with the study or study drug, whether or not it was considered to be related to the treatment. A serious AE (SAE) was defined as an $\mathrm{AE}$ that resulted in death, was life-threatening, required inpatient hospitalization or prolongation of existing hospitalization, resulted in a persistent or significant incapacity or substantial disruption of the ability to conduct normal life, resulted in birth defects in the offspring of the participant, or could lead to one of the previously listed outcomes without medical or surgical intervention. $\mathrm{AE}$ and laboratory data, including shifts to abnormal levels and trends in values over time, were evaluated in detail for toxicities seen with other subcutaneously or intravenously administered ASO drugs, including thrombocytopenia and hepatic and renal impairment [18-20], and for relationships to disorders associated with SMA, including cardiovascular abnormalities and respiratory illnesses [21-23], or the route of drug administration (e.g., post-lumbar puncture syndrome [PLPS]) [24, 25]. Clinical laboratory analyses were evaluated using analysis of shifts relative to the normal range from baseline to minimum (low) and maximum (high) post-baseline values. Shifts to low included individuals with normal, high, or unknown values at baseline and at least one post-baseline value on the given laboratory test. Shifts to high included individuals with low, normal, or unknown values at baseline and at least one post-baseline value on the given laboratory test. ECG qualitative results included an overall interpretation categorized as normal, abnormal but not clinically significant, or abnormal and clinically significant. Shifts from unknown or normal at baseline to abnormal and clinically significant or abnormal and not clinically significant during the post-baseline period were evaluated.

\subsection{Statistical Considerations}

Treatment duration (exposure to study treatment) was defined as the time from administration of the first dose to the last day of follow-up. Incidence rates of AEs were tabulated by Medical Dictionary for Regulatory Activities (MedDRA) System Organ Class and Preferred Term. The rate of PLPS was derived from the Preferred Term included under the injury, poisoning and procedural complications System Organ Class. Data including treatment duration, amount of study drug received, and incidence of AEs and SAEs were analyzed by treatment (nusinersen and control). Events related to post-lumbar puncture were analyzed on a per-procedure basis in the later-onset population using MedDRA Preferred Terms of back pain, cerebrospinal fluid leakage, headache, nausea, PLPS, procedural pain, procedural nausea, procedural headache, and vomiting.

\section{Results}

\subsection{Participants and Treatment Exposure}

Data were analyzed from 323 infants and children with symptomatic SMA, including 240 treated with nusinersen (100 with infantile-onset SMA and 140 with later-onset SMA) and 83 who underwent sham procedures (control: 41 infantileonset, 42 later-onset). Baseline characteristics are shown in Table 1 , and any differences generally reflect the individual study designs (e.g., geographical location of study sites, inclusion age). The overall median (range) time on study for all nusinersen-treated participants was 449.0 (6-1538) days, for a total of 375.9 participant-years of exposure (Table S1 in the Electronic Supplementary Material [ESM]).

\subsection{Safety Events and Laboratory and Examination Findings}

Overall, $96 \%$ (231/240) of participants treated with nusinersen and $99 \%(82 / 83)$ of control participants experienced at least one AE (Table 2). The incidence of SAEs in nusinersen-treated participants was lower than that in the control group for both infantile-onset (77\% in nusinersen-treated vs. 95\% in controltreated participants) and later-onset SMA (16\% vs. 29\%). The incidence of AEs with fatal outcomes was lower among nusinersen-treated versus control-treated participants (8\% vs. 19\%). None of the participants with later-onset SMA died while on study. The causes of death in the infantile-onset studies were primarily respiratory in nature (10/18 fatal events [56\%] in nusinersen-treated infants and 12/16 fatal events [75\%] in control-treated infants) or were cardiac related (two nusinersen [11\%], three control [19\%]) and were consistent with those typically observed for infantile-onset SMA [1]; hence, the events were considered to be unrelated to nusinersen by the study investigator. There were six AEs with $\mathrm{a} \geq 20 \%$ incidence among the pooled nusinersen-treated participants: pyrexia, upper respiratory tract infection, nasopharyngitis, vomiting, headache, and constipation. These AEs, with the exception of headache (nusinersen group: 53 [22\%]; control group: 3 [4\%]), occurred at similar frequencies in the control group. One AE (procedural nausea) was considered related to study drug by the investigator; $41 \mathrm{AEs}$ were considered possibly related, and those occurring in more than one participant included headache $(n=9)$, pyrexia $(n=8)$, back pain $(n=7)$, PLPS $(n=4)$, vomiting $(n=3)$, and tachycardia $(n=2)$.

\subsubsection{Respiratory Illness}

The overall incidence of respiratory, thoracic, and mediastinal disorders in participants treated with nusinersen was higher in those with infantile-onset SMA than in those with 
Table 1 Baseline demographics

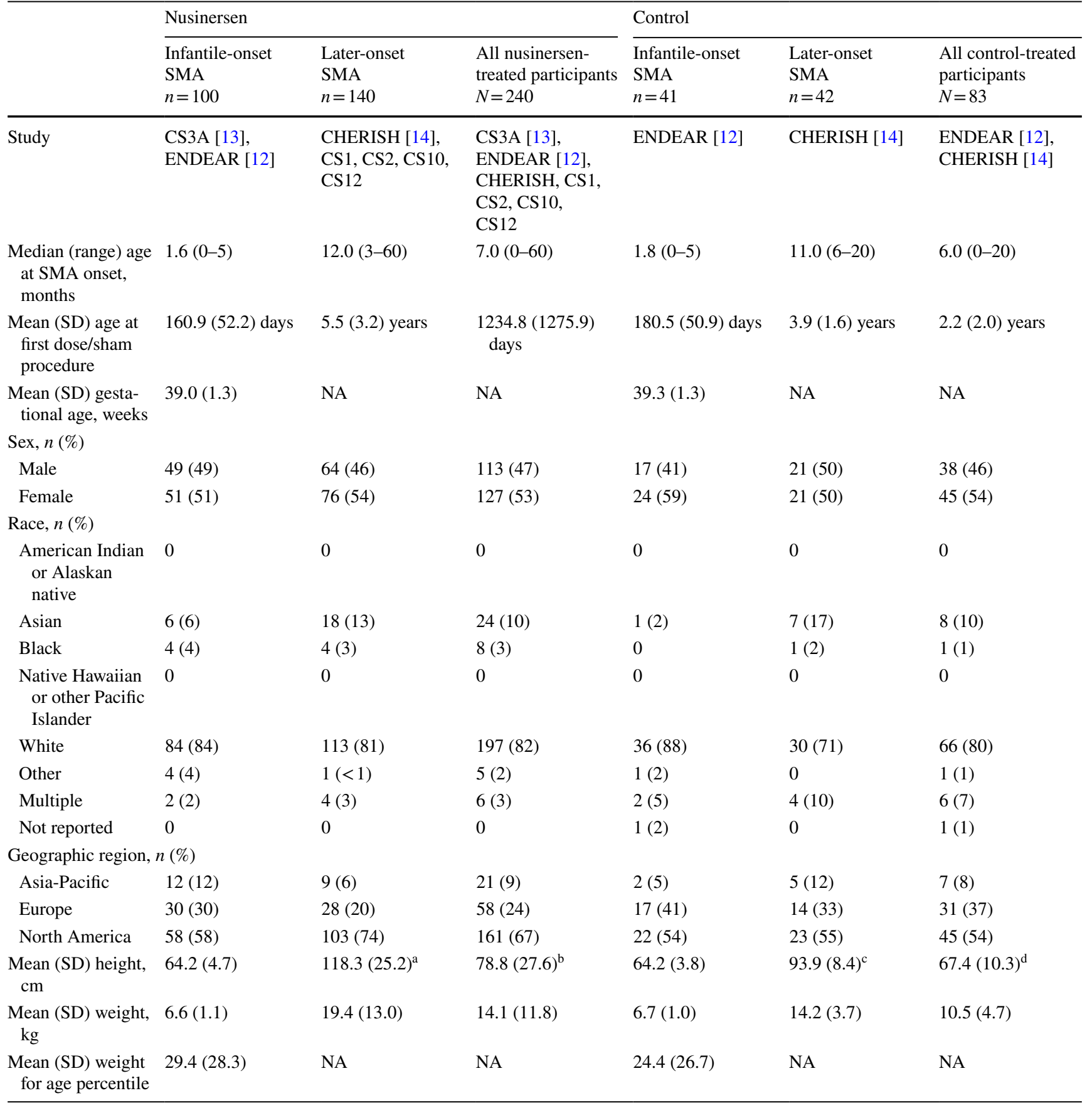

$N A$ not applicable, $S D$ standard deviation, SMA spinal muscular atrophy

${ }^{\mathrm{a}} n=37$

${ }^{\mathrm{b}} n=137$

${ }^{\mathrm{c}} n=5$

${ }^{\mathrm{d}} n=46$

later-onset SMA (79\% vs. $44 \%$, respectively; Table S2 in the ESM), and among the former group, incidence was slightly lower in those treated with nusinersen (79\%) compared with those who received control (88\%). Upper respiratory tract infections were common in all groups, regardless of nusinersen treatment: for participants with infantile-onset SMA and later-onset SMA, respectively, upper respiratory tract infection incidences were $22 \%$ and $45 \%$ for control 
Table 2 AE summary

\begin{tabular}{|c|c|c|c|c|c|c|}
\hline & \multicolumn{3}{|c|}{ Nusinersen } & \multicolumn{3}{|l|}{ Control } \\
\hline & $\begin{array}{l}\text { Infantile- } \\
\text { onset } \\
\text { SMA } \\
n=100\end{array}$ & $\begin{array}{l}\text { Later-onset } \\
\text { SMA } \\
n=140\end{array}$ & $\begin{array}{l}\text { All nusinersen- } \\
\text { treated } \\
\text { participants } \\
N=240\end{array}$ & $\begin{array}{l}\text { Infantile- } \\
\text { onset } \\
\text { SMA } \\
n=41\end{array}$ & $\begin{array}{l}\text { Later-onset } \\
\text { SMA } \\
n=42\end{array}$ & $\begin{array}{l}\text { All control- } \\
\text { treated } \\
\text { participants } \\
N=83\end{array}$ \\
\hline Any AE, $n(\%)$ & $97(97)$ & $134(96)$ & 231 (96) & $40(98)$ & $42(100)$ & 82 (99) \\
\hline \multicolumn{7}{|l|}{ Common AEs, $n(\%)^{\mathrm{a}}$} \\
\hline Pyrexia & $62(62)$ & $52(37)$ & $114(48)$ & $24(59)$ & $15(36)$ & $39(47)$ \\
\hline Upper respiratory tract infection & $36(36)$ & $54(39)$ & $90(38)$ & $9(22)$ & $19(45)$ & $28(34)$ \\
\hline Nasopharyngitis & $23(23)$ & $36(26)$ & $59(25)$ & $4(10)$ & $15(36)$ & $19(23)$ \\
\hline Vomiting & $22(22)$ & $36(26)$ & $58(24)$ & $8(20)$ & $5(12)$ & $13(16)$ \\
\hline Headache & $1(1)$ & $52(37)$ & $53(22)$ & 0 & $3(7)$ & $3(4)$ \\
\hline Constipation & $37(37)$ & $12(9)$ & $49(20)$ & $9(22)$ & $5(12)$ & $14(17)$ \\
\hline Cough & $16(16)$ & $27(19)$ & $43(18)$ & $8(20)$ & $9(21)$ & $17(20)$ \\
\hline Back pain & $1(1)$ & $40(29)$ & $41(17)$ & 0 & 0 & 0 \\
\hline Pneumonia & $31(31)$ & $9(6)$ & $40(17)$ & $7(17)$ & $8(19)$ & $15(18)$ \\
\hline Respiratory distress & $30(30)$ & $4(3)$ & $34(14)$ & $12(29)$ & $2(5)$ & $14(17)$ \\
\hline Scoliosis & $12(12)$ & $20(14)$ & $32(13)$ & $2(5)$ & $3(7)$ & $5(6)$ \\
\hline Joint contracture & $13(13)$ & $15(11)$ & $28(12)$ & $2(5)$ & $7(17)$ & $9(11)$ \\
\hline Respiratory failure & $27(27)$ & $1(<1)$ & $28(12)$ & $16(39)$ & $1(2)$ & $17(20)$ \\
\hline Diarrhea & $17(17)$ & $10(7)$ & $27(11)$ & $7(17)$ & $3(7)$ & $10(12)$ \\
\hline Post-lumbar puncture syndrome ${ }^{b}$ & 0 & $27(19)$ & $27(11)$ & 0 & 0 & 0 \\
\hline Viral infection & $13(13)$ & $13(9)$ & $26(11)$ & $3(7)$ & $2(5)$ & $5(6)$ \\
\hline Atelectasis & $24(24)$ & $1(<1)$ & $25(10)$ & $12(29)$ & 0 & $12(14)$ \\
\hline \multicolumn{7}{|l|}{ Severity of reported AEs, $n(\%)$} \\
\hline Mild & $9(9)$ & $52(37)$ & $61(25)$ & $1(2)$ & $19(45)$ & $20(24)$ \\
\hline Moderate & $31(31)$ & $72(51)$ & $103(43)$ & $6(15)$ & $20(48)$ & $26(31)$ \\
\hline Severe & $57(57)$ & $10(7)$ & $67(28)$ & $33(80)$ & $3(7)$ & $36(43)$ \\
\hline Incidence of serious AEs, $n(\%)$ & $77(77)$ & $22(16)$ & $99(41)$ & $39(95)$ & $12(29)$ & $51(61)$ \\
\hline Incidence of treatment-related AEs, $n(\%)$ & 0 & $1(<1)$ & $1(<1)$ & 0 & 0 & 0 \\
\hline $\begin{array}{l}\text { Incidence of possibly treatment-related } \\
\text { AEs, } n(\%)\end{array}$ & $12(12)$ & $29(21)$ & $41(17)$ & $6(15)$ & $4(10)$ & $10(12)$ \\
\hline $\begin{array}{l}\text { Incidence of AEs leading to treatment } \\
\text { discontinuation, } n(\%)\end{array}$ & $17(17)$ & 0 & $17(7)$ & $16(39)$ & 0 & $16(19)$ \\
\hline $\begin{array}{l}\text { Incidence of AEs with a fatal outcome, } n \\
(\%)\end{array}$ & $18(18)$ & 0 & $18(8)$ & $16(39)$ & 0 & $16(19)$ \\
\hline Incidence of death, $n(\%)$ & 18 (18) & 0 & $18(8)$ & $16(39)$ & 0 & $16(19)$ \\
\hline
\end{tabular}

$A E$ adverse event, $S M A$ spinal muscular atrophy

${ }^{\mathrm{a}} \geq 10 \%$ in nusinersen-treated participants

${ }^{\mathrm{b}}$ Preferred Term included under the injury, poisoning and procedural complications System Organ Class

participants and 36\% and 39\% for nusinersen-treated participants (Table 2). Respiratory distress and respiratory failure were relatively common among all nusinersen-treated participants (34 [14\%] and 28 [12\%], respectively), and were higher in those with infantile-onset SMA (30 [30\%] and 27 [27\%], respectively). Rates were similarly high in children with infantile-onset SMA who received sham procedure in ENDEAR (respiratory distress: 12 [29\%]; respiratory failure: 16 [39\%]). High rates of respiratory events were anticipated in participants with infantile-onset SMA given the baseline disease characteristics of both nusinersen-treated and control-treated infants in ENDEAR: SMA symptoms at baseline were experienced by higher proportions of participants in the nusinersen versus control group, and included paradoxical breathing ( $89 \%$ and $66 \%$ ), pneumonia or respiratory symptoms (35\% and 22\%), and swallowing or feeding difficulties (51\% and 29\%), while ventilatory support (use of any ventilation) was required by $26 \%$ and $15 \%$, respectively [12]. Other respiratory-related AEs occurring at high rates in nusinersen- and control-treated infantile-onset SMA 
participants included atelectasis (24\% vs. $29 \%$ ) and acute respiratory failure (17\% vs. $24 \%$; Table S2 in the ESM). The incidence of respiratory, thoracic, and mediastinal SAEs in nusinersen- and control-treated participants with infantileonset SMA was $63 \%$ and $73 \%$, respectively, and included respiratory distress (29\% and 20\%), respiratory failure (25\% and $39 \%)$, acute respiratory failure (17\% and $22 \%)$, atelectasis (16\% and $10 \%)$, aspiration pneumonia (11\% and $12 \%)$, and bronchial secretion retention ( $2 \%$ and $12 \%$ ).

In the ENDEAR study, the rate of hospitalization (adjusted annualized rate; 95\% confidence interval [CI]) was 4.328 (3.608-5.192) with nusinersen and 5.704 (4.392-7.408) with control; reasons for hospitalization were similar between groups, and respiratory-related events accounted for $59 \%$ of all hospitalizations in the nusinersentreated group and $64 \%$ of hospitalizations in the sham procedure-treated group [26]. The overall time spent hospitalized was lower in nusinersen-treated versus sham procedure-treated infants (least squares mean 0.114 vs. 0.207 ; least squares mean treatment difference - 0.093 [95\% CI -0.151 to -0.034$] ; p=0.0022$ ). Among later-onset participants, the incidence of respiratory thoracic and mediastinal SAEs was lower with nusinersen than with control (4\% vs. $7 \%)$.

\subsubsection{Cardiac Disorders and ECG Results}

Overall incidence of cardiac disorders was relatively low (13\% [32/240]) among all nusinersen-treated participants. The most common cardiac disorder in the nusinersen treatment groups was tachycardia, occurring in $10 \%(10 / 100)$ of infantile-onset SMA participants and 4\% (5/140) of later-onset SMA children, with a total incidence of $6 \%$ (15/240) among all nusinersen-treated children. Incidences of tachycardia were similarly low in control groups and were reported in 12\% (5/41) and 5\% (2/42) of participants with infantile- and later-onset SMA, respectively, with a total incidence of $8 \%$ (7/83). The incidence of clinically significant ECG shifts to abnormal was similar between all nusinersen-treated participants (5\% [10/190]) and all control-treated participants (3\% [2/67]; Table S3 in the ESM).

\subsubsection{Post-lumbar Puncture Syndrome (PLPS)}

Reported incidences of PLPS and events associated with PLPS [24, 25] were higher in nusinersen-treated children with later-onset SMA compared with participants with infantile-onset SMA who were younger and may have had limited ability to communicate these symptoms. PLPS was reported in 27 of $140(19 \%)$ participants with later-onset SMA who were treated with nusinersen: $11 \%$ reported mild events, $7 \%$ reported moderate events, and $<1 \%$ reported a severe event. PLPS, vomiting, headache, and back pain occurred in $19 \%$ versus $0 \%, 26 \%$ versus $22 \%, 37 \%$ versus $1 \%$, and $29 \%$ versus $1 \%$ of participants with later-onset versus infantile-onset SMA, respectively. The incidence of these events was also higher in nusinersen-treated than in control-treated participants who did not undergo the lumbar puncture procedure, with rates of $11 \%$ versus $0 \%$ for PLPS, $24 \%$ versus $16 \%$ for vomiting, $22 \%$ versus $4 \%$ for headache, and $17 \%$ versus $0 \%$ for back pain.

Post-lumbar puncture events were analyzed on a perprocedure basis in later-onset participants. In those treated with nusinersen (655 lumbar punctures), $13 \%$ and $21 \%$ of procedures were associated with an AE within $24 \mathrm{~h}$ and $168 \mathrm{~h}$, respectively, while in control participants (168 sham procedures), $3 \%$ of 168 sham procedures were associated with an $\mathrm{AE}$ at both time points. Ninety-three percent of all lumbar puncture procedures were performed with a 22- or 25 -gauge needle (needle size range 19 - to 27 -gauge). The median (range) duration of post-lumbar puncture events commencing within $72 \mathrm{~h}$ of the procedure was $2.0(1-106)$ days.

\subsubsection{Platelet Levels and Coagulation Abnormalities}

Similar proportions of participants demonstrated shifts to low platelets in the nusinersen-treated groups (ENDEAR 13\%; CHERISH 20\%; Table 3). The incidence of shifts to low platelets was higher among nusinersen-treated compared with control infants in ENDEAR (13\% vs. 0\%), but lower among nusinersen-treated patients compared with control children in CHERISH (20\% vs. $26 \%$ ). There were no sustained shifts to low platelets, and no AEs of thrombocytopenia were reported. There were no cases of sustained or severe thrombocytopenia in participants treated with nusinersen, nor were there any bleeding-related AEs associated with decreased platelet counts reported in the nusinersentreated population. Platelet counts $<20 \times 10^{9} / \mathrm{L}$ occurred in one infant in the ENDEAR study, two children in the CHERISH study, and none of the participants in CS3A and CS12. In the ENDEAR infant, the single low platelet count was preceded by a high count $\left(584 \times 10^{9} / \mathrm{L}\right)$ and followed by normal levels at all other assessments. In one of the two children from CHERISH, the single low platelet count was preceded and followed by all normal levels. In the second child from CHERISH, two isolated low platelet counts were recorded 245 days apart, with all other measurements demonstrating normal platelet levels. Median platelet counts were generally stable over time and were similar between nusinersen-treated and control-treated participants (Fig. 2).

\subsubsection{Renal Function Assessments}

The incidence of shifts to high blood urea nitrogen, high creatinine, or high cystatin $\mathrm{C}$ were low in each of the 
Table 3 Shifts to abnormal laboratory values

\begin{tabular}{|c|c|c|c|c|}
\hline \multirow[b]{2}{*}{ Parameter, $n / N(\%)$} & \multicolumn{2}{|c|}{ Infantile-onset SMA (ENDEAR) } & \multicolumn{2}{|c|}{ Later-onset SMA (CHERISH) } \\
\hline & $\begin{array}{l}\text { Nusinersen-treated } \\
N=80\end{array}$ & $\begin{array}{l}\text { Control-treated } \\
N=41\end{array}$ & $\begin{array}{l}\text { Nusinersen-treated } \\
N=84\end{array}$ & $\begin{array}{l}\text { Control-treated } \\
N=42\end{array}$ \\
\hline High blood urea nitrogen & $1 / 73(1)$ & $0 / 34$ & $2 / 84(2)$ & $1 / 42(2)$ \\
\hline High creatinine & $0 / 73$ & $0 / 34$ & $1 / 84(1)$ & $0 / 42$ \\
\hline High cystatin $\mathrm{C}$ & $1 / 73(1)$ & $1 / 33(3)$ & $1 / 83(1)$ & $0 / 42$ \\
\hline Urinary protein $\left(\geq 1 \text { positive }^{\mathrm{a}} \text { result }\right)^{\mathrm{b}}$ & $7 / 66(11)$ & $6 / 32(19)$ & $14 / 83(17)$ & 6/41 (15) \\
\hline 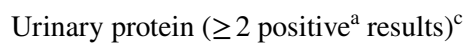 & $1 / 55(2)$ & $0 / 23$ & $1 / 83(1)$ & $1 / 41(2)$ \\
\hline Low platelets & $9 / 70(13)$ & $0 / 33$ & $15 / 76(20)$ & $10 / 39(26)$ \\
\hline High total bilirubin (direct) & $0 / 68$ & $0 / 30$ & $0 / 84$ & $0 / 42$ \\
\hline High total bilirubin (indirect) & $0 / 68$ & $0 / 28$ & $0 / 84$ & $1 / 42(2)$ \\
\hline High alkaline phosphatase & $3 / 72(4)$ & $0 / 32$ & $0 / 84$ & $0 / 42$ \\
\hline High ALT & $10 / 69(14)$ & 4/31 (13) & $3 / 82(4)$ & $4 / 42(10)$ \\
\hline High AST & $5 / 70(7)$ & $1 / 31(3)$ & $2 / 83(2)$ & $2 / 42(5)$ \\
\hline
\end{tabular}

$A L T$ alanine aminotransferase, $A S T$ aspartate aminotransferase

${ }^{\text {a } D e f i n e d ~ a s ~} \geq 1+$ on urine dipstick

${ }^{\mathrm{b}}$ The number at risk for shift to positive is the number of participants whose baseline values were not positive and who had at least one postbaseline lab assessment

${ }^{\mathrm{c}}$ The number at risk for shift to positive is the number of participants whose baseline values were not positive and who had at least two postbaseline lab assessments

nusinersen-treated groups $(0-2 \%)$ as well as the control groups (0-3\%; Table 3, Fig. 3). Similar proportions of nusinersen-treated participants demonstrated at least one positive urinary protein result (defined as $\geq 1+$ on urine dipstick) in ENDEAR and CHERISH (11\% and 17\%). Incidences of at least one positive urinary protein result were lower among nusinersen-treated compared with control infants in ENDEAR (11\% vs. 19\%) and similar in CHERISH (17\% vs. 15\%). Two nusinersen-treated children experienced two or more positive urinary protein results (Table 3); the positive results were separated by normal readings in one child and consecutive in the other child. The overall incidence of renal and urinary disorders AEs was low in all treatment groups (2-4\%; Table S2 in the ESM).

\subsubsection{Hepatic Function Assessments}

The incidence of shifts to high total bilirubin (direct), high total bilirubin (indirect), and high alkaline phosphatase were low among nusinersen- and control-treated infants/ children in the ENDEAR ( $0 \%$ vs. $0 \%, 0 \%$ vs. $0 \%$, and $4 \%$ vs. $0 \%$, respectively) and CHERISH studies (0\% vs. $0 \%$, $0 \%$ vs. $2 \%$, and $0 \%$ vs. $0 \%$, respectively; Table 3 ). No clear patterns in incidence of shifts to high alanine aminotransferase (ALT) or high aspartate aminotransferase (AST) were evident among participants with infantile-onset SMA and later-onset SMA (Table 3; Fig. S1 in the ESM). No Hy's Law cases were reported in nusinersen- and controltreated infants/children in the ENDEAR and CHERISH studies, and none of the participants had an AE of liver failure.

\subsubsection{Growth}

In ENDEAR, mean length/height and weight for age percentiles (percentile [standard deviation $\{\mathrm{SD}\}]$ ) were similar between nusinersen-treated and control infants with infantile-onset SMA at baseline (45.8 [33.42] vs. 40.8 [34.16] and 26.8 [27.81] vs. 24.4 [26.73], respectively). Mean length/ height for age percentiles decreased by $16.1 \%$ (SD 38.88; $n=26$ ) and increased by $18.6 \%$ (SD 22.41, $n=10$ ) in nusinersen-treated and control infants, respectively, by last assessment at day 394 in ENDEAR. Mean weight for age percentiles increased by $0.1 \%$ (SD 35.77, $n=26$ ) and increased by $20.7 \%$ (SD 29.60, $n=11$ ) in nusinersen-treated and control infants, respectively, by day 394 in ENDEAR. In CHERISH, mean (SD) weight was similar between nusinersen-treated and control infants with later-onset SMA at baseline (14.9 [4.78] kg vs. 14.2 [3.70] kg, respectively) and at last assessment at day $456(17.3$ [6.37] kg, $n=66$, vs. 16.9 [4.88] kg, $n=34$, respectively). 
Fig. 2 Median, minimum, and maximum platelet counts in nusinersen-treated and control participants from a ENDEAR and b CHERISH. Study visit day 2: nusinersen, $n=80$; control, $n=40$. $L L N$ lower limit of normal, $U L N$ upper limit of normal a

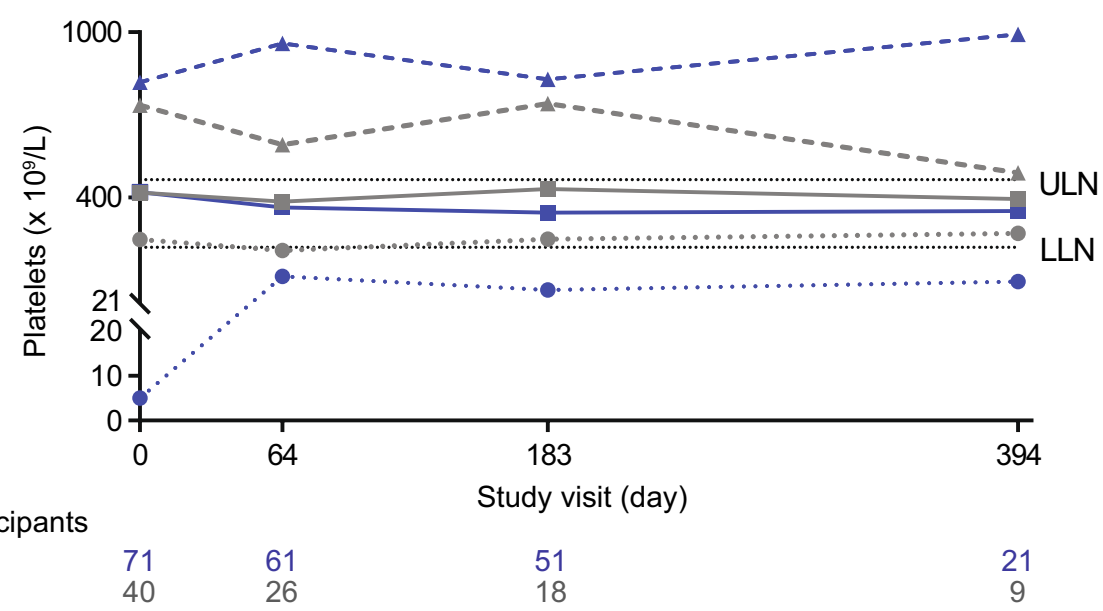

b

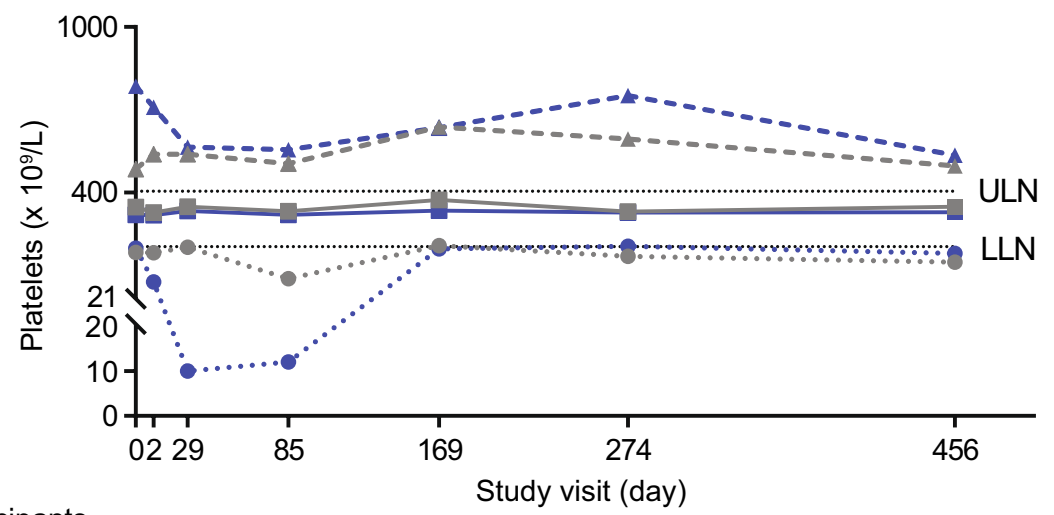

No. of participants

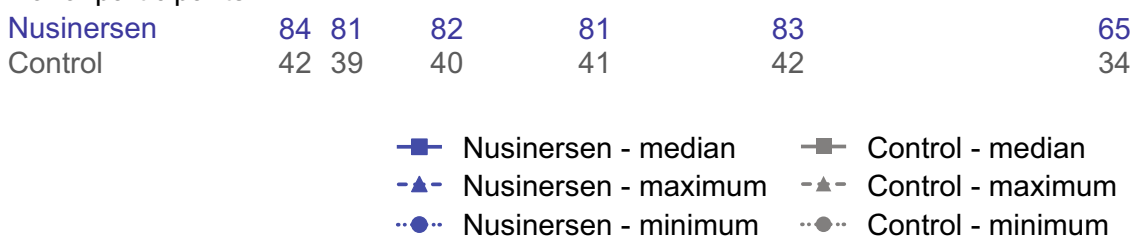

\section{Discussion}

This safety analysis of data from seven clinical trials of nusinersen in more than 300 infants and children with symptomatic SMA showed that the rates and types of AEs reported in those treated with nusinersen were consistent with symptoms of SMA or the lumbar puncture procedure [23-25, 27]. Incidences of respiratory illness were generally higher in the infantile-onset SMA groups (nusinersen-treated and control), who had more severe disease (SMA type I) than older children (later-onset; SMA type II or III). The relatively high rate of respiratory events was consistent with the natural history of SMA, as pulmonary disease represents the major cause of morbidity and mortality in individuals with SMA types I and II [23]. In the ENDEAR study, respiratoryrelated events accounted for the majority of hospitalizations in both the nusinersen-treated and sham procedure-treated groups [26]. All events that led to death occurred in the infantile-onset SMA group, but the overall incidence of such events was higher in control-treated infants compared with nusinersen-treated infants. Upon medical review by the site investigator and sponsor, the types of events leading to death were considered consistent with direct or indirect causes of death observed in the context of infantile-onset SMA. Participants in all studies received consistent standards of care including airway clearance and coughing assistance, respiratory support, and management of nutritional needs [23]; specifically, greater utilization of pulmonary, gastrointestinal, and orthopedic consultants and dieticians may have occurred in this study than in routine clinical care. Additionally, vaccination rates may have been higher among participants from the included studies than in other SMA populations due to the standard of care guidelines. Thus, SMA disease-related events may be even further decreased in both the nusinersen 
Fig. 3 Median, minimum, and maximum creatinine levels in nusinersen-treated and control participants from a ENDEAR and $\mathbf{b}$ CHERISH. Study visit day 2: nusinersen, $n=81$; control, $n=40$. $L L N$ lower limit of normal, $U L N$ upper limit of normal a

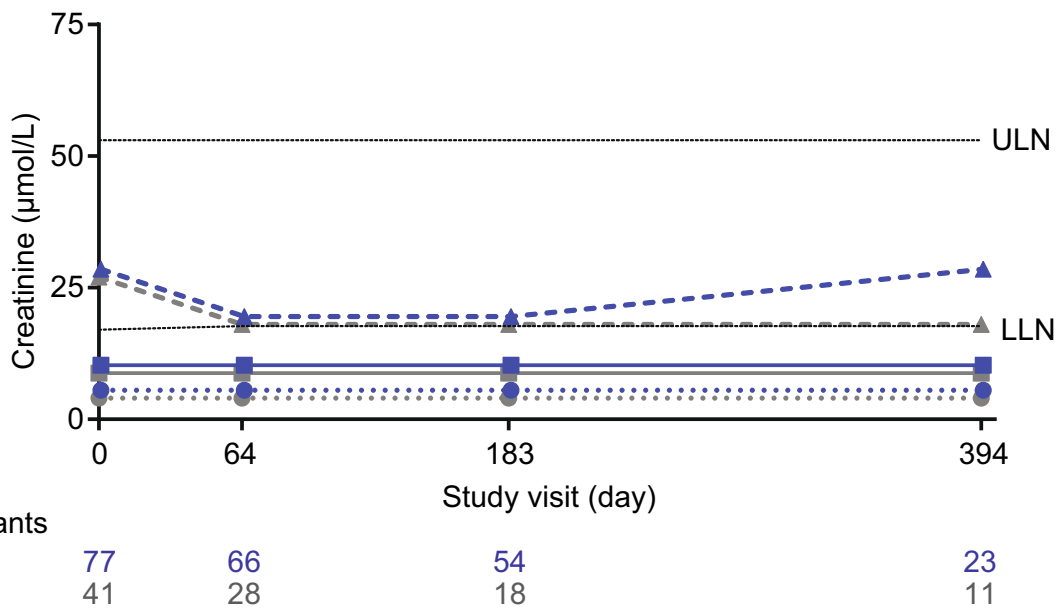

b

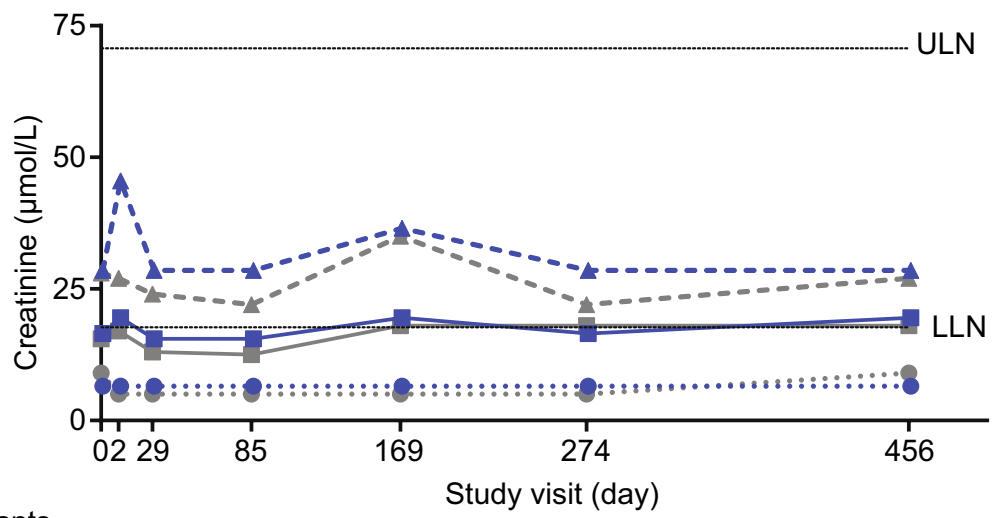

No. of participants

Nusinersen

8482

82

83

84

66

Control

4241

41

41

34

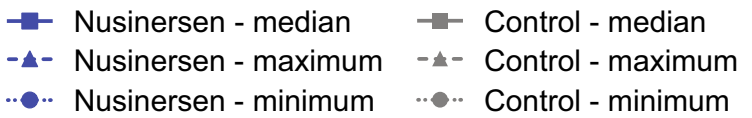

and control arms compared with the general SMA community, where clinical care may be more varied than in the studies included in this analysis [28-30].

PLPS-associated events, including headache, back pain, and vomiting, were more common in older participants (later-onset SMA) than in infants. This could be because the younger participants with infantile-onset SMA were not yet verbal and thus could not report these lumbar puncture-related symptoms. Incidences of events associated with PLPS have been shown to be more common in children $\geq 10$ years old compared with those $<10$ years old and are less common in children than adults [24]. The overall rates of post-lumbar events when analyzed on a per-procedure basis (13\% and $21 \%$ after 24 and $168 \mathrm{~h}$, respectively) were comparable to rates of $16-27 \%$ for headache and $40 \%$ for back pain reported elsewhere in children who undergo lumbar puncture procedures [24, 31]. None of the headaches reported in these patients were identified by the site investigator as an indication of increased intracranial pressure or hydrocephalus.

Mean weight for age percentile remained stable in nusinersen-treated infants from baseline to the day 394 assessment, while an increase in mean weight for age percentile over this time period was observed in control infants. The larger weight increase in control infants over the course of the study could be related to differences in caloric demands and motor function between groups. For instance, control infants may have reduced caloric demands with progressive weakness, compared with increasing caloric demands and motor function in those treated with nusinersen. Another explanation for the differences observed could be the challenge in estimating caloric requirements for both groups of infants. A larger percentage of control versus nusinersentreated infants underwent gastrointestinal tube placement during the course of the study (41\% vs. $30 \%$ ). A gastrointestinal tube allows additional calories to be provided, 
even if not necessary. Mean length/height for age percentile decreased in nusinersen-treated infants and increased in control infants from baseline to the day 394 assessment. The mechanisms by which nusinersen could affect weight and height are not known. However, the differences in height between nusinersen-treated and control infants are most likely due to measurement challenges (e.g., hyptonia, contractures) or measurement errors. Infants with poor muscle tone would measure longer than those with more muscle tone whose limbs would not be fully extended during the measurement. Weight is closely linked to height, and overweight children are taller on average than non-overweight children [32], indicating that the increased incidence of gastrointestinal tube placement to facilitate nutrition and feeding in the control group could also have led to the increased length gain in that group.

A range of toxicities associated with ASOs have been reported in human clinical trials and include increased aminotransferase levels (e.g., ALT and AST), proteinuria, and thrombocytopenia [20,33]. Although often referred to as class effects, these toxicities are only seen with a minority of ASOs tested in humans [34-36]; they appear to result from the chemical structure of the ASOs and the individual nucleic acid sequence, are related to dose (i.e., systemic exposure), and are usually self-limiting after dosing is stopped [20, 33, 37, 38]. In some cases they may represent a unique interaction between drug and disease [37], and in other cases they may relate to the targeted pathway rather than to the ASO itself $[39,40]$. Renal and liver injury most likely occur due to accumulation with repeated doses [38], because subcutaneously or intravenously administered ASOs bind to plasma proteins that are filtered at the glomerulus and then reabsorbed in the proximal tubule [38, 41]. Proteinuria may be a sign of either glomerular or tubular toxicity [38]. Although ASO-associated glomerulonephritis is uncommon, the presence of high molecular weight proteins or large increases of proteins in the urine may be indicative of glomerulonephritis [38]. While thrombocytopenia has been one of the major concerns in the development of some ASO-based therapies, the underlying mechanism is still under investigation and may differ by severity and species [38, 42-44].

In the current integrated safety analysis, there was no systemic evidence of the types of toxicities that have been reported with some ASOs, including thrombocytopenia and hepatic and renal impairment [18-20]. This may be in part because nusinersen differs from other ASOs in its route of administration (intrathecal vs. subcutaneous or intravenous), dose, and dosing frequency; therefore, a lower level of systemic exposure is expected. No cases of severe, sustained thrombocytopenia were reported in nusinersen-treated participants. The US prescribing information recommends monitoring of urine protein concentration, platelet count, and coagulation at baseline and prior to each administration of nusinersen, and monitoring platelet counts and coagulation as clinically needed [45]. Repeat testing and further evaluation is recommended for urine protein concentrations $>0.2 \mathrm{~g} / \mathrm{L}$. No imbalance in the incidence of AEs signifying possible liver dysfunction was noted between nusinersen-treated participants and those who received sham procedure, and none of these AEs were reported as serious; all events resolved. Median laboratory values of direct bilirubin, indirect bilirubin, alkaline phosphatase, ALT, and AST were similar between groups and remained stable over time. There were no reports of renal failure, glomerulonephritis, nephrotic syndrome, or other relevant renal toxicity in participants receiving nusinersen. The results reported here for shift to positive urinary protein are semi-quantitative and based on the definition of a result of $\geq 1+$ on urine dipstick, which is approximately equivalent to levels of $0.3 \mathrm{~g} / \mathrm{L}$ [46]. The occurrence of proteinuria is similar to or lower than that reported previously [45] and is not suggestive of renal toxicity, given the relative stability of blood urea nitrogen, creatinine, and cystatin C levels.

Preliminary safety data on nusinersen use in real-world clinical practice have been reported by several groups [47-50]. In one report based on the experience from seven centers in Germany in children with infantile-onset SMA, SAEs were reported in $54.7 \%$ of treated children, severe AEs in $45.3 \%$, and PLPS in 4.9\% [48]. The most frequently observed AEs across the reports were respiratory events, fever, headache, nausea, and vomiting [47-50]. With the exception of a transient decrease in platelet count in one patient with mild thrombocytopenia at treatment initiation, there were no relevant changes in clinical laboratory values in 28 adults with SMA Type II or III treated with nusinersen [49]. Although collection and reporting of safety parameters varied in the real-world reports, the safety profile of nusinersen observed in real-world clinical practice is consistent with that observed in the current integrated analysis of clinical study data.

There are limitations to this safety analysis. For data from ENDEAR and CHERISH, due to early study termination after positive interim analyses showing a benefit:risk profile favoring nusinersen $[12,14]$, not all children were assessed up to the final visit date. Therefore, participants at final assessment may not be representative of the whole cohort. Some AEs requiring verbalization from the participant (e.g., back pain, headache) may not have been captured in children who were not yet able to speak (i.e., those with infantile-onset SMA). This analysis only covers safety data from clinical trials and does not address potential findings from the post-market setting. Finally, due to small population sizes, rare AEs may not have been captured. Infants and 
children included in this safety analysis had the option to enroll in SHINE, which is collecting long-term safety data in these SMA populations.

\section{Conclusion}

Across the nusinersen clinical trial program in participants with symptomatic infantile-onset or later-onset SMA, nusinersen demonstrated a favorable safety profile, and most AEs and SAEs were consistent with the nature and frequency of events typically seen with SMA or in the context of the lumbar puncture procedure. No new safety findings emerged from this dataset $(N>300)$, and it represents 375.9 participant-years of exposure to nusinersen. These data, together with the significant and clinically meaningful benefits on motor function seen in infants and children with symptomatic SMA [12-16], support the early treatment of children with SMA.

Acknowledgements The authors would like to thank the children who participated in these studies and their parents/guardians, and all investigators and staff involved in the nusinersen clinical trials.

Data Availability Requests for data supporting this article should be submitted to the Biogen Clinical Data Request Portal (http://www. biogenclinicaldatarequest.com).

\section{Compliance with Ethical Standards}

Ethical Approval All study designs were approved by institutional review boards at study sites and were conducted in accordance with Good Clinical Practice guidelines.

Informed Consent Written informed consent was obtained from parents or legal guardians, and based on institutional guidelines and the child's age, participants provided assent as appropriate.

Funding These studies were sponsored by Ionis Pharmaceuticals, Inc. (Carlsbad, CA, USA) and Biogen (Cambridge, MA, USA). Biogen also provided funding for the open access fee and medical writing support in the development of this paper. Meryl Mandle and Allison Green from Excel Scientific Solutions wrote the first draft of the manuscript based on input from authors, and Nathaniel Hoover from Excel Scientific Solutions copyedited and styled the manuscript per the journal requirements. Biogen and Ionis Pharmaceuticals, Inc. reviewed and provided feedback on the manuscript to the authors. The authors had full editorial control of the manuscript and provided their final approval of all content.

Conflict of interest Basil T. Darras has served on advisory boards for AveXis, Biogen, Cytokinetics, Dynacure, Genentech, PTC, Roche, and Sarepta; served on a speakers bureau for Biogen; received research support from the National Institutes of Health/National Institute of Neurological Disorders and Stroke, the Slaney Family Fund for SMA, the SMA Foundation, and the Working on Walking Fund; received grants from Biogen, CureSMA, and Ionis Pharmaceuticals, Inc. during ENDEAR, CHERISH, CS2, CS11, and CS12; received grants from Cytokinetics, FibroGen, PTC, Roche, Santhera, Sarepta, and Summit; and has no personal financial interests in these companies. Michelle A. Farrar has received scientific advisory board honoraria from Biogen and Roche; is a principal investigator for ongoing AveXis and Biogen clinical trials; and has received funding from Motor Neuron Diseases Research Institute of Australia. Eugenio Mercuri has served on advisory boards for SMA studies for AveXis, Biogen, Ionis Pharmaceuticals, Inc., Novartis, and Roche; is a principal investigator for ongoing Ionis Pharmaceuticals, Inc./Biogen and Roche clinical trials; and has received funding from Famiglie SMA Italy, Italian Telethon, and SMA Europe. Richard S. Finkel has received grants and advisor fees from Biogen and Ionis Pharmaceuticals, Inc. during ENDEAR and CHERISH; received grants from AveXis, Cytokinetics, and Roche; served as an advisor to AveXis, Novartis, and Roche outside the submitted work; served in an advisory capacity to nonprofit organizations Cure SMA, SMA Europe, the SMA Foundation, and SMA Reach (UK); and served on a data safety monitoring board for the AveXis AVXS-101 phase 1 gene transfer study and the Roche Moonfish phase 1b study; received fees for presentations at CME activities sponsored by AveXis and Biogen; and has received licensing fees from the Children's Hospital of Philadelphia for co-creating the CHOP INTEND motor scale for SMA. Richard Foster is an employee of and stockholder in Biogen. Steven G. Hughes was an employee of and holds stock/stock options in Ionis Pharmaceuticals, Inc. He now consults for Ionis Pharmaceuticals, Inc. Ishir Bhan is an employee of and stockholder in Biogen. Wildon Farwell is an employee of and stockholder in Biogen. Sarah Gheuens is an employee of and stockholder in Biogen.

Open Access This article is distributed under the terms of the Creative Commons Attribution-NonCommercial 4.0 International License (http://creativecommons.org/licenses/by-nc/4.0/), which permits any noncommercial use, distribution, and reproduction in any medium, provided you give appropriate credit to the original author(s) and the source, provide a link to the Creative Commons license, and indicate if changes were made.

\section{References}

1. Darras BT, Monani UR, De Vivo DC. Genetic disorders affecting the motor neuron: spinal muscular atrophy. In: Swaiman KF, Ashwal S, Ferriero DM, Schor NF, Finkel RS, Gropman AL, et al., editors. Swaiman's pediatric neurology: principles and practice. 6th ed. Edinburgh: Elsevier; 2017. p. 1057-64.

2. Arkblad E, Tulinius M, Kroksmark AK, Henricsson M, Darin N. A population-based study of genotypic and phenotypic variability in children with spinal muscular atrophy. Acta Paediatr. 2009;98(5):865-72.

3. Jedrzejowska M, Milewski M, Zimowski J, Zagozdzon P, KosteraPruszczyk A, Borkowska J, et al. Incidence of spinal muscular atrophy in Poland-more frequent than predicted? Neuroepidemiology. 2010;34(3):152-7.

4. Lunn MR, Wang CH. Spinal muscular atrophy. Lancet. 2008;371(9630):2120-33.

5. Prior TW, Snyder PJ, Rink BD, Pearl DK, Pyatt RE, Mihal DC, et al. Newborn and carrier screening for spinal muscular atrophy. Am J Med Genet Part A. 2010;152A(7):1608-16.

6. Prior TW. Spinal muscular atrophy: a time for screening. Curr Opin Pediatr. 2010;22(6):696-702.

7. Finkel R, Bertini E, Muntoni F, Mercuri E. 209th ENMC International Workshop: Outcome Measures and Clinical Trial Readiness in Spinal Muscular Atrophy 7-9 November 2014, Heemskerk, The Netherlands. Neuromuscul Disord. 2015;25(7):593-602. 
8. Finkel RS, Sejersen T, Mercuri E. 218th ENMC International Workshop: revisiting the consensus on standards of care in SMA Naarden, The Netherlands, 19-21 February 2016. Neuromuscul Disord. 2017;27(6):596-605.

9. Darras BT, Markowitz JA, Monani UR, De Vivo DC. Spinal muscular atrophies. In: Darras BT, Jones HRJ, Ryan MM, De Vivo DC, editors. Neuromuscular disorders of infancy, childhood, and adolescence: a clinician's approach. 2nd ed. San Diego: Academic Press; 2014. p. 117-45.

10. Mercuri E, Finkel RS, Muntoni F, Wirth B, Montes J, Main M, et al. Diagnosis and management of spinal muscular atrophy: part 1: recommendations for diagnosis, rehabilitation, orthopedic and nutritional care. Neuromuscul Disord. 2018;28(2):103-15.

11. Hua Y, Sahashi K, Hung G, Rigo F, Passini MA, Bennett CF, et al. Antisense correction of SMN2 splicing in the CNS rescues necrosis in a type III SMA mouse model. Genes Dev. 2010;24(15):1634-44.

12. Finkel RS, Mercuri E, Darras BT, Connolly AM, Kuntz NL, Kirschner J, et al. Nusinersen versus sham control in infantile-onset spinal muscular atrophy. $\mathrm{N}$ Engl $\mathrm{J}$ Med. 2017;377(18):1723-32.

13. Finkel RS, Chiriboga CA, Vajsar J, Day JW, Montes J, De Vivo DC, et al. Treatment of infantile-onset spinal muscular atrophy with nusinersen: a phase 2, open-label, dose-escalation study. Lancet. 2016;388(10063):3017-26.

14. Mercuri E, Darras BT, Chiriboga CA, Day JW, Campbell C, Connolly AM, et al. Nusinersen versus sham control in later-onset spinal muscular atrophy. N Engl J Med. 2018;378(7):625-35.

15. Darras BT, Chiriboga CA, Iannaccone ST, Swoboda KJ, Montes J, Mignon L, et al. Nusinersen in later-onset spinal muscular atrophy: long-term results from the phase $1 / 2$ studies. Neurology. 2019;92(21):e2492-506.

16. Chiriboga CA, Swoboda KJ, Darras BT, Iannaccone ST, Montes J, De Vivo DC, et al. Results from a phase 1 study of nusinersen $\left(\right.$ ISIS-SMN $\mathrm{Rx}_{\mathrm{R}}$ ) in children with spinal muscular atrophy. Neurology. 2016;86(10):890-7.

17. Haché M, Swoboda KJ, Sethna N, Farrow-Gillespie A, Khandji A, Xia S, et al. Intrathecal injections in children with spinal muscular atrophy: nusinersen clinical trial experience. J Child Neurol. 2016;31(7):899-906.

18. Panta R, Dahal K, Kunwar S. Efficacy and safety of mipomersen in treatment of dyslipidemia: a meta-analysis of randomized controlled trials. J Clin Lipidol. 2015;9(2):217-25.

19. Shimizu-Motohashi Y, Murakami T, Kimura E, Komaki H, Watanabe N. Exon skipping for Duchenne muscular dystrophy: a systematic review and meta-analysis. Orphanet J Rare Dis. 2018;13(1):93.

20. Chan JHP, Lim S, Wong WSF. Antisense oligonucleotides: from design to therapeutic application. Clin Exp Pharmacol Physiol. 2006;33(5-6):533-40.

21. Rudnik-Schöneborn S, Heller R, Berg C, Betzler C, Grimm $\mathrm{T}$, Eggermann $\mathrm{T}$, et al. Congenital heart disease is a feature of severe infantile spinal muscular atrophy. J Med Genet. 2008;45(10):635-8.

22. Hamilton G, Gillingwater TH. Spinal muscular atrophy: going beyond the motor neuron. Trends Mol Med. 2013;19(1):40-50.

23. Wang CH, Finkel RS, Bertini ES, Schroth M, Simonds A, Wong $\mathrm{B}$, et al. Consensus statement for standard of care in spinal muscular atrophy. J Child Neurol. 2007;22(8):1027-49.

24. Ebinger F, Kosel C, Pietz J, Rating D. Headache and backache after lumbar puncture in children and adolescents: a prospective study. Pediatrics. 2004;113(6):1588-92.

25. Morgenlander JC. Lumbar puncture and CSF examination. Postgrad Med. 1994;95(8):125-31.

26. Tulinius M, Bruno C, Deconinck N, Schara U, Finkel RS, Bhan I, et al. Nusinersen decreases the incidence and length of hospitalizations in infants with spinal muscular atrophy: results from the ENDEAR study. In: SMA Europe-1st International Scientific Congress on Spinal Muscular Atrophy, Krakow, Poland, 25-27 January 2018.

27. Oskoui M, Darras BT, De Vivo DC. Spinal muscular atrophy: 125 years later and on the verge of a cure. In: Sumner CJ, Paushkin S, Ko CP, editors. Spinal muscular atrophy. New York: Academic Press; 2017. p. 1-18.

28. Hardart MK, Burns JP, Truog RD. Respiratory support in spinal muscular atrophy type I: a survey of physician practices and attitudes. Pediatrics. 2002;110(2):e24.

29. Benson RC, Hardy KA, Gildengorin G, Hsia D. International survey of physician recommendation for tracheostomy for spinal muscular atrophy type I. Pediatr Pulmonol. 2012;47(6):606-11.

30. Oskoui M, Ng P, Liben S, Zielinski D. Physician driven variation in the care of children with spinal muscular atrophy type 1 . Pediatr Pulmonol. 2017;52(5):662-8.

31. Crock C, Orsini F, Lee KJ, Phillips RJ. Headache after lumbar puncture: randomised crossover trial of 22-gauge versus 25-gauge needles. Arch Dis Child. 2014;99(3):203-7.

32. Júlíusson PB, Brannsether B, Kristiansen H, Hoppenbrouwers K, Bjerknes R, Roelants M. Should children with overweight or obesity be excluded from height references? Arch Dis Child. 2015;100(11):1044-8.

33. Jason TL, Koropatnick J, Berg RW. Toxicology of antisense therapeutics. Toxicol Appl Pharmacol. 2004;201(1):66-83.

34. Crooke ST, Baker BF, Kwoh TJ, Cheng W, Schulz DJ, Xia S, et al. Integrated safety assessment of 2'-O-methoxyethyl chimeric antisense oligonucleotides in nonhuman primates and healthy human volunteers. Mol Ther. 2016;24(10):1771-82.

35. Crooke ST, Baker BF, Pham NC, Hughes SG, Kwoh TJ, Cai D, et al. The effects of 2'-O-methoxyethyl oligonucleotides on renal function in humans. Nucleic Acid Ther. 2018;28(1):10-22.

36. Crooke ST, Baker BF, Witztum JL, Kwoh TJ, Pham NC, Salgado $\mathrm{N}$, et al. The effects of 2 '- $O$-methoxyethyl containing antisense oligonucleotides on platelets in human clinical trials. Nucleic Acid Ther. 2017;27(3):121-9.

37. Crooke ST, Witztum JL, Bennett CF, Baker BF. RNA-targeted therapeutics. Cell Metab. 2018;27(4):714-39.

38. Frazier KS. Antisense oligonucleotide therapies: the promise and the challenges from a toxicologic pathologist's perspective. Toxicol Pathol. 2015;43(1):78-89.

39. Hashemi N, Odze RD, McGowan MP, Santos RD, Stroes ES, Cohen DE. Liver histology during mipomersen therapy for severe hypercholesterolemia. J Clin Lipidol. 2014;8(6):606-11.

40. Kastelein JJ, Wedel MK, Baker BF, Su J, Bradley JD, Yu RZ, et al. Potent reduction of apolipoprotein B and low-density lipoprotein cholesterol by short-term administration of an antisense inhibitor of apolipoprotein B. Circulation. 2006;114(16):1729-35.

41. Bennett CF, Swayze EE. RNA targeting therapeutics: molecular mechanisms of antisense oligonucleotides as a therapeutic platform. Annu Rev Pharmacol Toxicol. 2010;50:259-93.

42. Henry SP, Narayanan P, Shen L, Bhanot S, Younis HS, Burel SA. Assessment of the effects of 2'-methoxyethyl antisense oligonucleotides on platelet count in cynomolgus nonhuman primates. Nucleic Acid Ther. 2017;27(4):197-208.

43. Narayanan P, Shen L, Curtis BR, Bourdon MA, Nolan JP, Gupta S, Hoffmaster Ch, Zhou F, Christian B, Schaubhut JL, Grennlee S, Burel SA, Witztum JL, Engelhardt JA, Henry SP. Investigation into the mechanism(s) that leads to platelet decreases in cynomolgus monkeys during administration of ISIS 104838, a 2'-MOE-modified antisense oligonucleotide. Toxicol Sci. 2018;164(2):613-26.

44. Shen X, Corey DR. Chemistry, mechanism and clinical status of antisense oligonucleotides and duplex RNAs. Nucleic Acids Res. 2018;46(4):1584-600. 
45. Biogen. Spinraza [prescribing information]. 2018 [cited 3 June 2019]. https://www.accessdata.fda.gov/drugsatfda_docs/label /2018/209531s003s004lbl.pdf.

46. Carroll MF, Temte JL. Proteinuria in adults: a diagnostic approach. Am Fam Physician. 2000;62(6):1333-40.

47. Aragon-Gawinska K, Seferian AM, Daron A, Gargaun E, Vuillerot $\mathrm{C}$, Cances $\mathrm{C}$, et al. Nusinersen in patients older than 7 months with spinal muscular atrophy type 1 : a cohort study. Neurology. 2018;91(14):e1312-8.

48. Pechmann A, Langer T, Schorling D, Stein S, Vogt S, Schara U, et al. Evaluation of children with SMA type 1 under treatment with nusinersen within the expanded access program in Germany. J Neuromuscul Dis. 2018;5(2):135-43.

49. Stolte B, Totzeck A, Kizina K, Bolz S, Pietruck L, Monninghoff $\mathrm{C}$, et al. Feasibility and safety of intrathecal treatment with nusinersen in adult patients with spinal muscular atrophy. Ther Adv Neurol Disord. 2018;11:1756286418803246.

50. Sansone VA, Albamonte E, Salmin F, Casiraghi J, Pirola A, Bettinelli $\mathrm{M}$, et al. Intrathecal nusinersen treatment for SMA in a dedicated neuromuscular clinic: an example of multidisciplinary and integrated care. Neurol Sci. 2019;40(2):327-32.

\section{Affiliations}

\section{Basil T. Darras ${ }^{1} \cdot$ Michelle A. Farrar $^{2} \cdot$ Eugenio Mercuri $^{3} \cdot$ Richard S. Finkel $^{4} \cdot$ Richard Foster $^{5} \cdot$ Steven G. Hughes ${ }^{6}$. Ishir Bhan ${ }^{7}\left({ }^{1}\right)$. Wildon Farwell ${ }^{7}$. Sarah Gheuens ${ }^{7}$}

1 Department of Neurology, Boston Children's Hospital and Harvard Medical School, Boston, MA, USA

2 Sydney Children's Hospital Network and UNSW Medicine, UNSW Sydney, Sydney, Australia

3 Department of Paediatric Neurology, Catholic University, Rome, Italy
4 Division of Neurology, Department of Pediatrics, Nemours Children's Hospital, Orlando, FL, USA

5 Biogen, Maidenhead, Berkshire, UK

6 Ionis Pharmaceuticals, Inc., Carlsbad, CA, USA

7 Biogen, Cambridge, MA, USA 